\title{
The Arts of the Possible
}

Information Visualization in the Field of Politics

\section{Florian Windhager}

Department for Knowledge and Communication Management, Danube University Krems,

Dr.-Karl-Dorrek-Str. 30, A-3500 Krems, Austria, florian.windhager@donau-uni.ac.at, +4327328932338

\author{
Michael Smuc \\ Department for Knowledge and Communication Management, Danube University Krems, \\ Dr.-Karl-Dorrek-Str. 30, A-3500 Krems, Austria, florian.windhager@donau-uni.ac.at
}

\begin{abstract}
Information visualization offers multiple methods for making sense of complex data using graphic representations. These complement verbal representations and show rich potential for supporting cognition and communication in numerous areas of application, including the field of political communication and education. Yet - and despite a strong increase in options with regard to the accessibility of data, tools, and methods - no conceptual framework or discussion has as yet sought to organize these emerging visual vocabularies and their possible (re-)combinations. Against this background, we discuss the layout principles of existing visualization methods and look to align them within a coherent framework that allows a multimodal navigation of modern news and information spaces. In doing so, we also consider relevant ways and means of minimizing well-known barriers in the public and political communication realm.
\end{abstract}

Keywords: Information visualization, politics, public communication, methods, visual literacy

A complex collaborative endeavors, modern societies thrive by trading and sharing vast varieties of goods, responsibilities, and problem solving skills - as long as they can keep their productive patterns of collaboration and competition from turning into combat and commotion. While conflict management is ensured by the enforcement of laws, the means to develop and issue these common rules are assigned to the specific field of politics. To control associated risks of abuse, democracies expect their members to frequently evaluate their political institutions - and to do so from an at least vaguely informed position regarding their aims and operations. To ensure knowledge transfer on such aims and operations, entire professions - from teachers and journalists to spokespersons and lobbyists - work to communicate between the field of politics and the public. In doing so, they are challenged both by a non-trivial subject matter on the one side and an unknown diversity of interests, prior knowledge, attention, and motivations on the other. Given that such a complex scenario calls and demands the advancement and refinement of communication methods, we seek to rethink its classical challenges by reflecting on recent developments in the visual communication realm.

Information Visualization (InfoVis) complements traditional means of language-based communication and plays an increasing role in media, science, and education. Its rise in use is accompanied by a consolidation of this field of practice in academia, where it is defined as the study of how to effectively present information visually or - with a more practical focus on modern media - as "the use of computer-supported, interactive, visual representations of abstract data to amplify cognition" (Card, Mackinlay, \& Shneiderman, 1999, p.8). Thus promising "to help us speed our understanding and action in a world of increasing information volumes" (Card 2008, p.542), InfoVis aims to provide insights into complex subject matters for experts as well as "for the people" (Danziger, 2008). 
The "standard rationale" in this developing field (van Wijk, 2005) - with its contention that most of its methods can help to empower people in multiple ways - makes it especially interesting when it comes to politics, a topic which has been deemed to concern "us all" ever since reflections on the interdependencies of private and public life emerged in ancient times. Providing the basic vocabulary for a visual language, InfoVis applications already support numerous communication efforts on the part of individual mediators (like teachers, researchers, or journalists) and institutions (e.g., governments, political parties, interest groups, news media, etc.). But like traditional, language-based approaches to communication, information visualization as a cultural technique requires careful attention and appropriate training. If these are ensured, it would enable news and facts to be graphically encoded and decoded on the common sender and receiver side, and allow for deeper understanding of visual representations in numerous expert fields and professions.

The motivation behind this article lies in the fact that - despite the increasing amount of visual representations already encountered in society - visual literacy as a prerequisite for their qualified production and interpretation (cf. Felten, 2008) remains a rare form of conscious knowledge, resulting predominantly only from individual reflection efforts or further education. While various standard methods are frequently used to illustrate political facts and figures, broader reflections on their conceptual implications and interconnections are still lacking. Existing political textbooks neither reflect on their visual vocabularies, nor do they leverage possible synergies within larger visual analytical frameworks. Accordingly, the field of politics will continue to encode the lion's share of its information using spoken or written language, requiring its listeners and readers to deal with ever extending sequences of information verbalization and heightened cognitive loads. Against this background, it seems legitimate to question to what extent the worrisome phenomenon of political apathy might be constantly co-produced by the equally worrisome phenomenon of poor information design, i.e. not only by a lack of interest on the part of the recipients, but also by a lack of engaging graphical representations, which would enrich the essential concepts and discussions of political speech and writing and allow them to unfold on the canvas of a second (perceptual and cognitive) modality.

Far from claiming to be a systematic elaboration, this article seeks simply to propose a first scaffold for organizing the discussion and practice of political information visualization. We begin in section 1 by outlining a generic setup within which visual communication should take place. Section 2 introduces some basic methods of political information visualization and arranges them in a synergistic conceptual framework. While this section aims to overcome visual communication barriers by discussing these basic methods and the connections between them, section 3 discusses further strategies to tackle common obstacles between the senders and 'the people', reframed in this case as an audience of non-experts who primarily receive information in casual contexts.

\section{A Visual Communication Model}

As with the use of language in general, communication by visual means has to be located and conceptually analyzed as something that happens between at least two cognizing agents. Despite the potential of such communication models to spark far-reaching conceptual discussions (cf. Risku, Mayr, Windhager, \& Smuc, 2011), we seek to make use of such an extended representation to better organize the discussion surrounding visual communication and information design (see Fig. 1). With regard to the common sender-(message)-receiver-axis, we refer in this article to anyone who gives form to data (e.g., teachers, journalists, politicians) as an "information designer" (A) and place the "public" (B) as a heterogeneous group of recipients on the other side. This axis is extended by two other components, namely a "subject matter", which provides the topic of communication, and the corresponding "data" which is to be brought into shape in the form of an "InfoVis artifact" (i.e. images on paper or screens). "Information visualization methods" do so in a standardized way, aiming to inform and serve the "public" (B) with their specific "goals and motivations". To successfully complete this chain (providing B with insights into the subject matter), various "communication barriers" have to be overcome - especially with regard to an non-expert intended audience. As a compendium of procedures to overcome such barriers, the function of 
"usability methods" is to raise the communication success ratio - as we will discuss in more detail below.

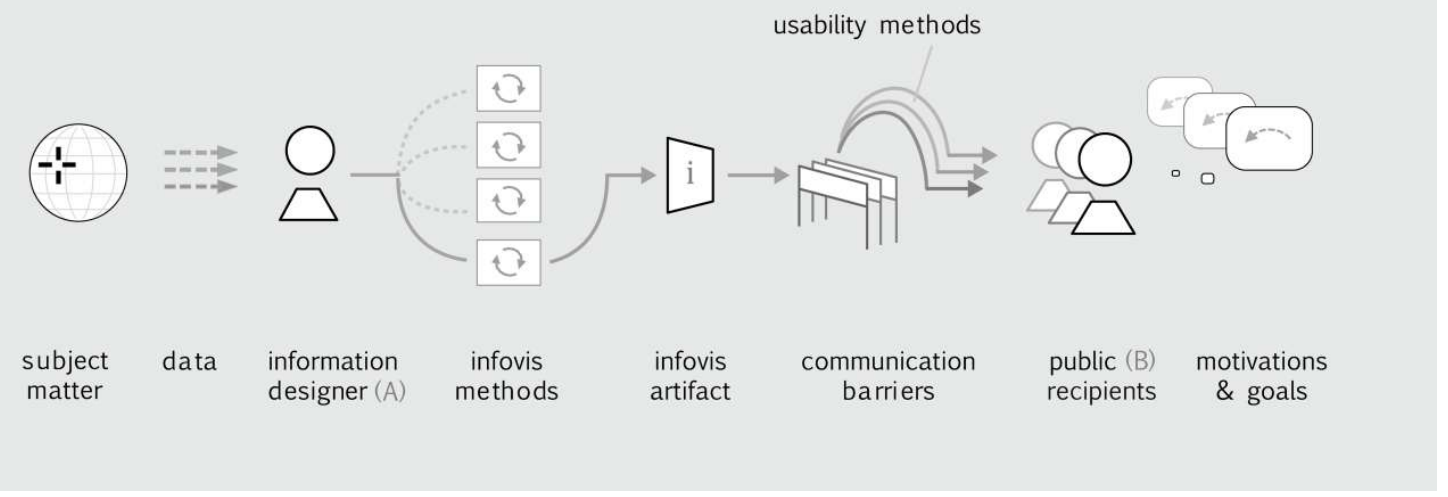

Figure 1: A Visual Communication Model ${ }^{1}$

The descriptions provided below follow a problem- and methods-centered approach and elaborate on one of the first and most basic challenges in visual communication: Given the field of political topics and data - which visualization methods can communicators (A) make use of - and should recipients $(B)$ be able to make sense of? Since they are located in the middle of the communication model, visualization methods literally determine the shapes that are given to a subject matter, and thus co-produce the original object by predefining the look of the visual artifact. The communicator has to make substantial design decisions here, and making sufficient sense of these decisions again requires a certain level of method literacy on the part of the receiver.

While information visualization as a research field focuses on advancing the arsenal of visualization methods (optimizing existing and developing new ones), its application in media and communication (e.g., in journalism or education) is more concerned with challenges of usability and understanding, beginning with the clarification of the basic operating principles behind such methods. In such a context, some of the first things that need to be known are: Which methods does the InfoVis toolkit offer? How do they work? What are the possibilities and limits of the different imaging procedures? How do the multiple information artifacts relate to each other as pieces of the larger political mosaic? Can we relocate them within a coherent framework, so that they not only illuminate individual bits and pieces, but also unveil visual synergies and shed light on each other?

\section{Political Information Visualization Methods}

From a communication model perspective (cf. Fig. 1), information designers traditionally choose a suitable visualization method by looking at the subject matter and data (left) and at the recipients and their motivations (right) and then selecting one or more of the available visualization methods for implementation. But what happens if a field does not as yet have a consolidated toolbox, and instead assembles and borrows its methods from neighboring fields? In such a case, the conceptual exploration and documentation of available methods will have to serve as a starting point for discussion. Accordingly, in the subsequent sections, we will rethink the methods that could enrich and fill the space between the maps and data visualization charts that readers of political coverage are used to encountering. In doing so, we will take a look in each case at how the method works and which insights it offers. Ultimately, we will touch upon how the different methods connect and translate to each other on a conceptual level, and on how a (re-)combination of their respective

${ }^{1}$ All figures in this article have been prepared and produced by the authors. 
results could technically be used to construct the whole political stage - from its regional to its extended, global scenery. In concrete terms, we will first look in detail at the physical map, political map, cartogram, bubble chart, network graph, word cloud, statistical data visualization, dynamic visualization, and political infographic methods, and then rewire them on a conceptual level.

\subsection{Physical and Political Maps}

Physical maps have serve as a revered visualization method to support the cognition of political agents since antiquity. The operating layout principle in this method can essentially be rephrased as: "Draw a selected environment from above." The result is a down-scaled representation, which shows the ground on which groups of individuals (from smaller packs to large federations) struggle for life, liberty, and the pursuit of happiness, and on which they face the frequent challenges which demand social coordination or collective decisions on how to deal with 'domestic' and 'foreign' affairs (Figure 2, left). The benefit of such maps lies in the fact that they illustrate where resources, risks, threats, chances for expansion, etc. are located in relation to a person's (or group's) own position, thus enabling them to navigate (plan, calculate, control, document, etc.) in unfamiliar territory. As such, the multitude of available examples stretches from the manuscript maps used in ancient times (Bagrov \& Skelton, 2009) to the zoomable satellite eyes encountered in the present day ( $r$ GoogleMaps).

Political maps go a step further by inscribing the (purported) borders of political entities - from small cities (gr. polis) to huge empires - into the physical environment. They also commonly use coloring or shading to incorporate further differentiations (Figure 2, right). The result shows the shapes of political territories as sovereign segments of the sociosphere, enriched by additional information on selected variables of political relevance. The benefits of such maps include the possible insights they offer into distributions of values (such as the existence of goods and resources), population densities, defense budgets, etc. Examples with frequently updated data range from the maps offered by Le Monde diplomatique (2012) to the $r$ WorldFactbookDashboard.
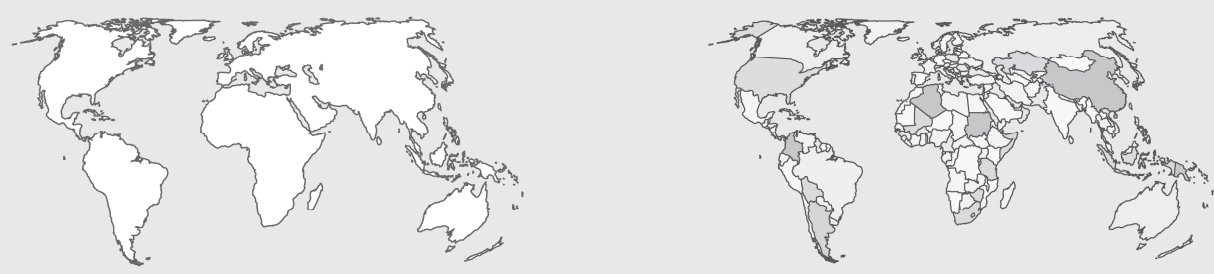

Figure 2: Examples of a physical map (left) and a political map (right), with the latter using various colors to visualize the extensions and possible attributes of political units.

\subsection{Cartograms}

While physical and political maps preserve the shapes and relative sizes of territories, the layout principle behind cartograms is the adaptation or distortion of areas according to a selected variable. In the case of non-contiguous cartograms, the results roughly maintain the locations of political entities, but deliberately resolve their neat arrangement. One particularly interesting layout option is the one used in Dorling cartograms (Dorling, 2011), where territories are uniformly represented as circular areas whose diameters depend on a selected variable (Figure 3, left). 
Benefits: By standardizing their shapes, cartograms provide an enhanced comparison view of political entities (e.g., every complex unit is a circle in Dorling cartograms) and use size variations to saliently show relevant differences. Examples: $>$ NYTimes; $>$ Mappingworlds.

\subsection{Bubble Charts}

While cartograms keep political entities near their geographic coordinates, bubble charts (and later network graphs) lift them up and re-arrange them according to new spatial layout principles driven by selected data (thus crossing the border from scientific to information visualization). The layout principle behind bubble charts can hence be rephrased as follows: "Erase the geographic grid and substitute it with a Cartesian plane, where an entity's size and its position on the $x$ - and $y$ axis is established by three of its intrinsic attributes." For example, the x-axis could show income per person, the $y$-axis life expectancy, and the size of each bubble the number of citizens (Fig. 3 , right).

Benefits: Similar to scatter plots, bubble charts allow us to visually analyze global distributions, identify clusters, and gain insights from single positions. Examples: rGapminder, $>$ WorldbankDataVisualizer.
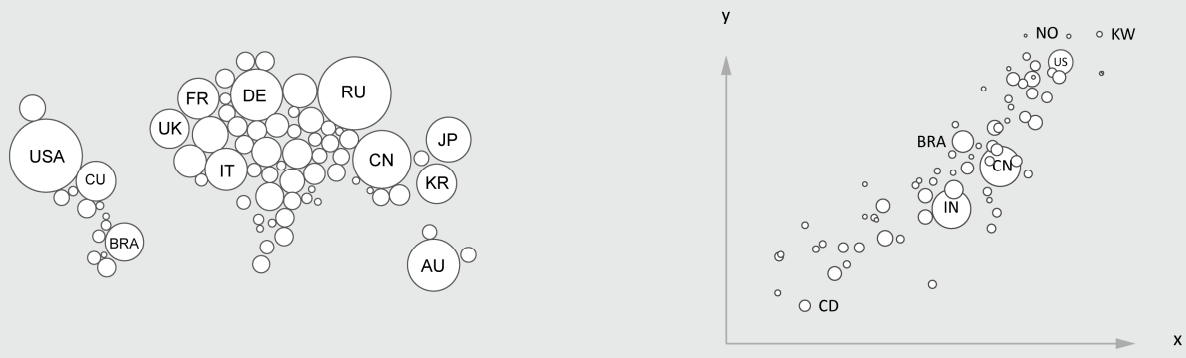

Figure 3: Examples of a cartogram (left) and a bubble chart (right), with the latter showing income per person ( $\mathrm{x}$-axis), life expectancy (y-axis), and number of citizens (bubble size).

\subsection{Network Graphs}

In contrast to bubble charts, network graphs (or node-link diagrams) arrange political entities according to selected empirical data on the relations between them. If any type of relation is given (e.g., flows of trade, air traffic, or bilateral agreements), the layout principle is often provided by socalled spring embedder algorithms, which execute the instructions: "Draw together nodes with strong relations and separate less connected nodes." The benefits of the resulting graphs are the emergence of data-driven topologies and relational clusters, and the possibility to localize single entities within them. The newly emerging proximities and distances between various entities provide insights into the structure of the social space that is created by economic or diplomatic relations (collaboration or conflict) or by the flows of capital, information, emotions, people, or goods through transport and media systems. Figure 4 (left) illustrates this imaging procedure using international trade data (adapted from $>$ Krempel\&Plümper). With their unrestricted visual granularity options, network graphs can represent social constellations from the macro-level (international networks), through the meso-level (institutional and inter-organizational networks), to the micro-level (social tissue, woven by inter-individual relations). Aside from spring embedder layouts, numerous alternate layouts methods can also govern the arrangement of nodes and links. A prominent example here are organizational charts, which are used to visualize the hierarchical 
relations within organizations and administrations. By reconstructing the major fields of a society (such as the economy, science, law, sports, health, religion, arts, etc.) as network layers with specific functions, governments become visible as managing networks of networks (see Fig. 7) engaged in the open-ended endeavors of controlling conflicts and promoting collective welfare within the (never undisputed) limits of the possible.

Semantic networks complement the representation of social networks and can cover all possible relations between conceptual or theoretical entities. By replacing social nodes with words or concepts, they provide the possibility to visually represent political structures and associated knowledge. Figure 4 (right), for example, illustrates the concept of "welfare" or "well-being" in a formalized way as the interplay of 11 distinct dimensions (OECD, 2013; rOECDBetterLife). Further examples here include $>$ Visualcomplexity, $>$ Relationbrowser.
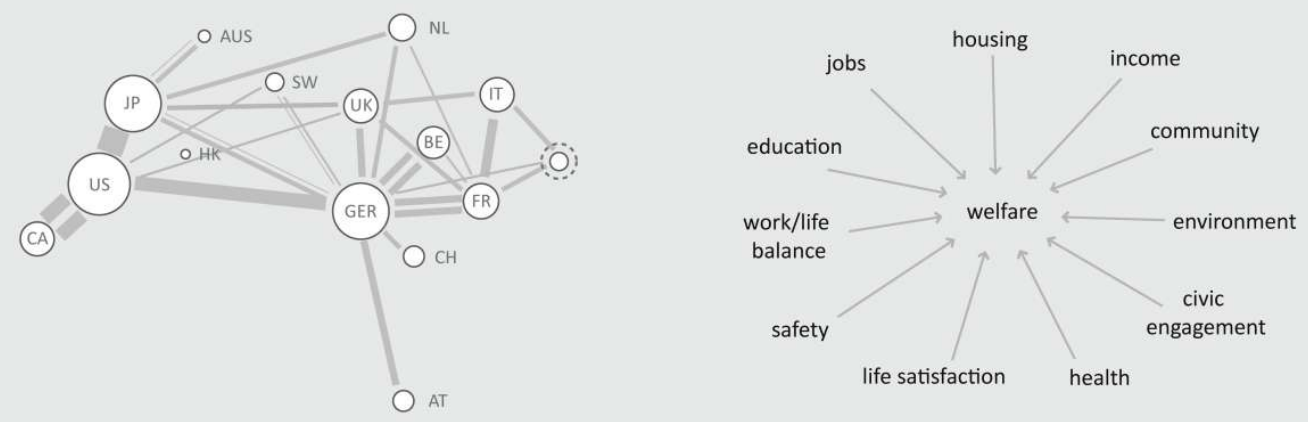

Figure 4: Examples of a social network graph visualizing flows of international trade (left) and a semantic network graph (cause-effect-diagram) displaying the relational concept of "welfare" (right).

\subsection{Word Clouds}

Social network graphs do not commonly pay any closer attention to the contents flowing through their links, despise the fact that the very existence of social ties essentially depends on the composition of these invisible streams of messages (e.g., in our case, from the verbal sequences in diplomatic letters and political speeches to the back and forth in discussions or controversies and the tides of "public opinion"). One method of offering a basic visual analysis of such complex content is word clouds (also known as tag clouds or weighted lists). Their layout principle reads: "Take the most important (key)words in any text or message and use the font size to display their frequency of use."

Benefits: Word clouds offer quick visual analyses of central themes, key issues, or foci of discussion (see Fig. 5, left). Furthermore, they are an accessible method of bridging from the InfoVis realm (which is often dependent on quantifiable (meta)data) to the pervasive realm of verbal representations with their complex semantic loads and intricate rhetorics (on which politics are usually exclusively dependent). Examples: $>$ InauguralSpeeches, $>$ Wordle.

\subsection{Statistic Data Visualization Methods}

With the exception of political maps, graphs and charts to visualize statistical data are the most widespread visualization methods used in the field of political communication. When addressed as a whole, no singular layout principle can be offered here, but the levels of visual literacy and documentation encountered (how to interpret bar, pie or line charts, etc.) are among the highest and most proficient. (e.g. Few, 2004). Benefits: As a comprehensive toolkit, such graphs and charts help to visually analyze the diverse types of quantitative data gathered or accumulated in every 
political entity. Since modern political systems process all their decisions (e.g. policy directions, options, scenarios, and the composition of their governing bodies) by the formation of majorities, it comes as no surprise that diagrams which show distributions (e.g., pie or ring charts) or provide precise quantitative comparisons (e.g., bar charts) are among the most widely used options when it comes to the coverage of public opinions and elections. Aside from these usual suspects, a range of advanced methods (e.g., radar charts, cf. Fig. 5) is also frequently used to gain insights into the complexities of socio-economic population data or to prepare, support, and evaluate political decision making. Examples: $>$ Visualizing, $>$ UNdata, $>$ GooglePublicData.
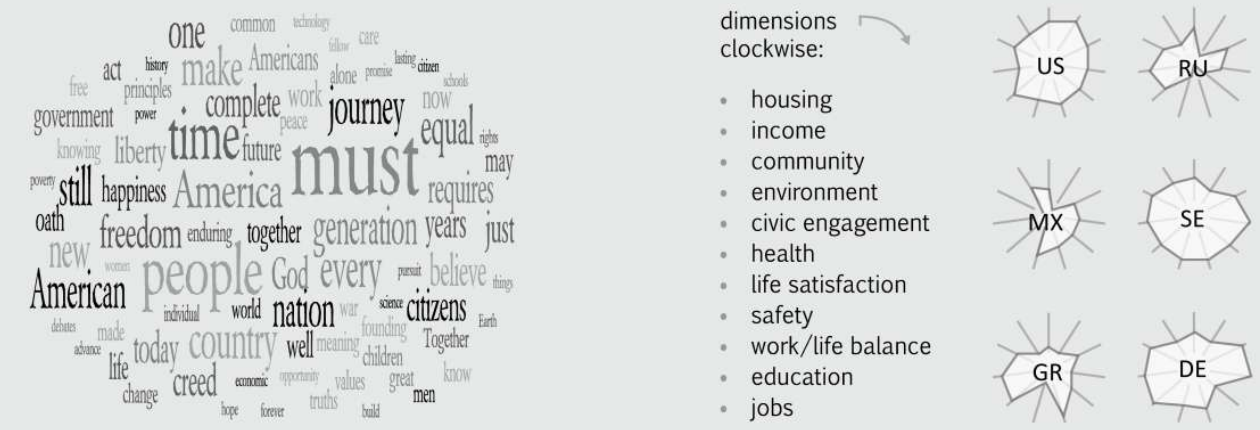

Figure 5: Examples of a word cloud for a political speech (left) and a statistical radar chart (right), visualizing the OECD Better Life Index for six different countries (cf. $>$ Visualizing).

\subsection{Dynamic Visualization Methods}

A challenge to all kinds of visualization methods poses time-oriented data about any short or long term dynamics of depicted constellations. Common solutions (see Figure 6) used to upgrade any given method on static data carriers like paper include the juxtaposition of temporal snapshots (Fig. 6, far left) or the superimposition of temporal layers with different line colors denoting different temporal states (Fig. 6, second from left). If we switch from paper to the screen, animation can also be used to display temporal change, e.g. by altering or changing an image (Fig. 6, second from right). Likewise, 2.5D layouts can be used to stack temporal layers and show temporal trajectories in the vertical space (Bach et al, 2014; Windhager, 2013) (Fig. 6, right).

A standard way of coping with the challenges of visual complexity is the implementation of interaction methods, which help to reduce the overall information in an image by only showing specific data on demand (see Section 3.3). By making users part of the imaging procedure, the explication and exploration of a complex data set becomes user driven, and thus cognitive operations (like the comparison of shapes or different points in time) are visually supported on the recipient's side.

\subsection{Political Infographics}

As a kind of "assembling meta-method", infographics allow the synthesis of any visual and verbal representation to illustrate a selected subject matter. Their simple layout principle ("Draw things together.") allows the creation of well-defined mashups of images, diagrams, and texts. 
Benefits: As an open genre, the benefits of infographics lie in their ability to selectively combine all the other visualization methods that have been available so far in a tailored framework that can be used to communicate any selected topic and can be enriched with explanatory text.

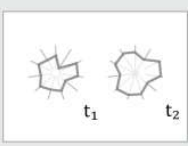

juxtaposition

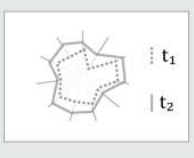

superimposition

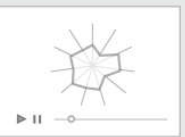

animation

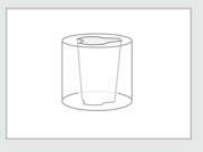

2.5 D layout

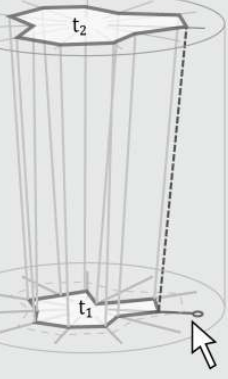

+ interaction

Figure 6: Different methods of visualizing change over time (from $t_{1}$ to $t_{2}$ ), including juxtaposition, superimposition, animation, and 2.5D layout, with the close up illustrating the supporting role of interaction methods.

Infographics can, for example, be used to shed light on complex constitutional architectures and governmental structures. Such structures are developed to ensure a state's basic functions providing the law and promoting welfare - while ideally correcting themselves through the separation of powers into an executive, a legislative, and a judicial level (see Fig. 7). Since social macro-units like nation states are heavily organized on multiple layers into different areas of activity (e.g., the economy, science, technology, education, religion, arts, media, etc.), their governing bodies have to differentiate correspondingly and are spread across multiple ministries (on the executive level) or extensive volumes of law (on the judicial level).

With nation states consisting of millions of actors who are all interwoven by different networks with different functions, a government (as the executive branch of a political system) has to be visualized as a managing "network of networks". This network collects resources from its citizens (taxation, Fig.7, bottom left) and uses them to re-assign problem solving procedures via its ministries to any affected areas and matters of concern (governance, Fig. 7, second from left) (cf. $r$ Death \& Taxes). Together with a representative body of non-governing actors (i.e. congressional or parliamentary opposition), the legislative function of this network is to create and amend laws by forging the necessary coalitions and obtaining the required majorities (legislation, Fig.7, center). The resulting bodies of law are interpreted and enforced by the judicial bodies (jurisdiction, Fig. 7 , second from right), which serve to manage conflicts and control any public or private activities. In contrast to other forms of government, democracies establish a self-organizing, cybernetic feedback cycle through the institution of regular elections, which serve as a form of bottom-up evaluation to confirm or deselect the current powers that be (election, Fig. 7, bottom right). ${ }^{2}$

\footnotetext{
${ }^{2}$ As democratic systems revolve around the formation and maintenance of majorities, they necessarily generate a prominent role for statistical data visualizations (cf. section 2.6), whose strength lies in their ability to render large quantities of data cognitively digestible. Mediated by colored pie or bar charts, which provide information on the status of shifting coalitions, contemporary citizens can now watch the composed procedures of populations exercising power by and on themselves - as long as they agree with the notion that immersing themselves as invisible pixels into a pie is worth the effort.
} 

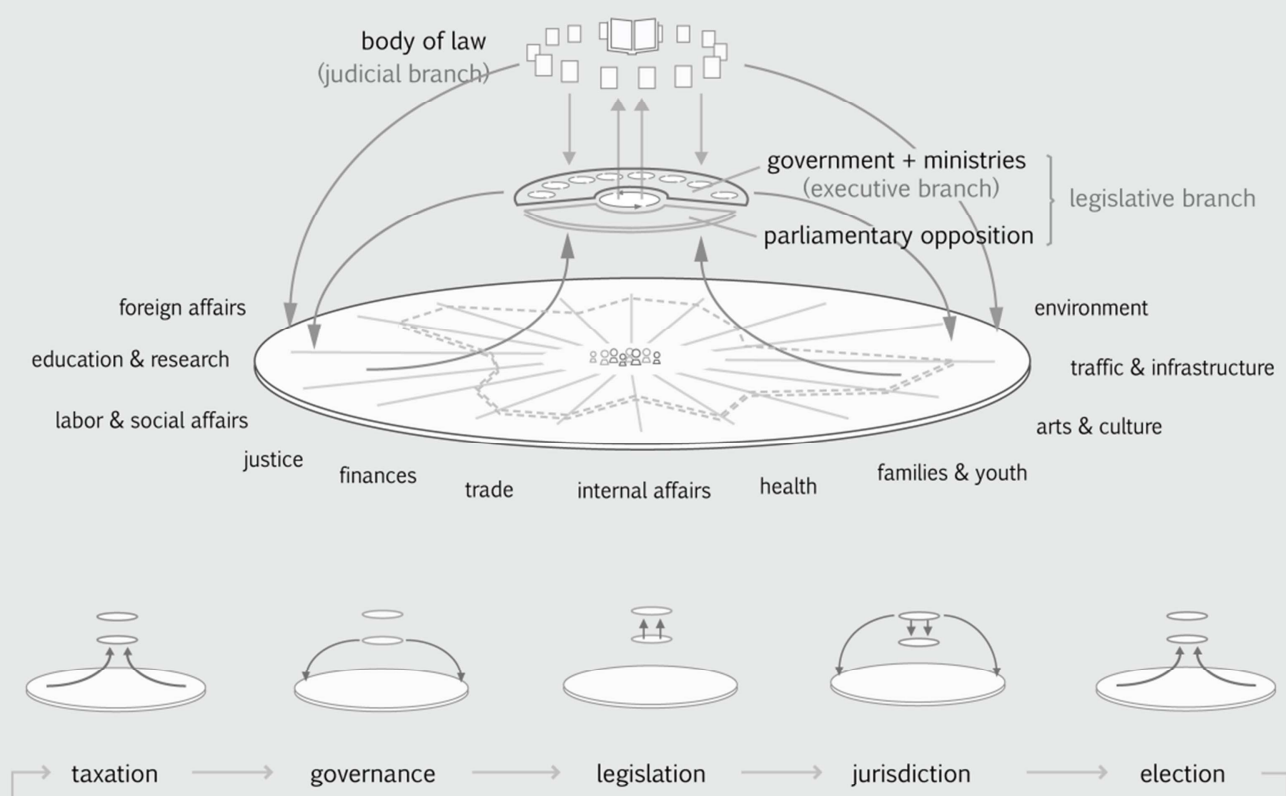

Figure 7: Political infographic, illustrating the political system of a democratic state based on the separation of powers. With all resources provided by the people (taxation), governments (executive branch) are managing 'networks of networks', provide problem solving solutions for matters of public concern (governance), develop laws (legislation) in conjunction with representative bodies (legislative branch), and are controlled by the judiciary, which also provides jurisdiction to the people, who in turn frequently evaluate the actors in all these institutions (elections).

\subsection{On the Interoperability of Visualization Methods}

All the methods presented above have already been applied by political communicators in various contexts. Yet one of the most promising - and hitherto unexploited - options would be not to treat such visualizations merely as isolated illustrations, but to systematically connect them using conceptual alignments, parallel arrangements, and visual transitions. Benefits: By doing so, the visualizations would shed light on each other by reciprocally sharing their visual vocabulary and connecting to each other in the form of "hyper-images". As such, they would allow coherent overviews to blend with detailed views on individual relevant components and pieces of the mosaic. Various visualization methods would then serve as complementary layouts for complex data, disclosing themselves as varying but interoperable perspectives which focus on the same subject matter from different distances and angles. ${ }^{3}$ From a cognitive science perspective, this allows a coherent "mental map" (Freire \& Rodríguez, 2006) to be plotted and expanded incrementally. From a semiotic point of view, such an approach taps into the visual-syntactic potential of the methods (Engelhardt, 2006), treating them like verbal sentences or propositions, which could be connected to produce higher levels of representational complexity.

\footnotetext{
${ }^{3}$ As a challenge for every visual multi-perspective framework, the transitions from information visualization methods to the visualization methods of photography and film require further consideration. This also applies for cross-border relations between scientific or documentary imaging methods (which claim not only empirical validity but also methodological transparency, cf. Dörk, Feng, Collins, \& Carpendale, 2013), for procedures from the realms of political spin and propaganda, as well as in the fine arts, where graphical representation motifs are not driven by knowledge or data alone.
} 


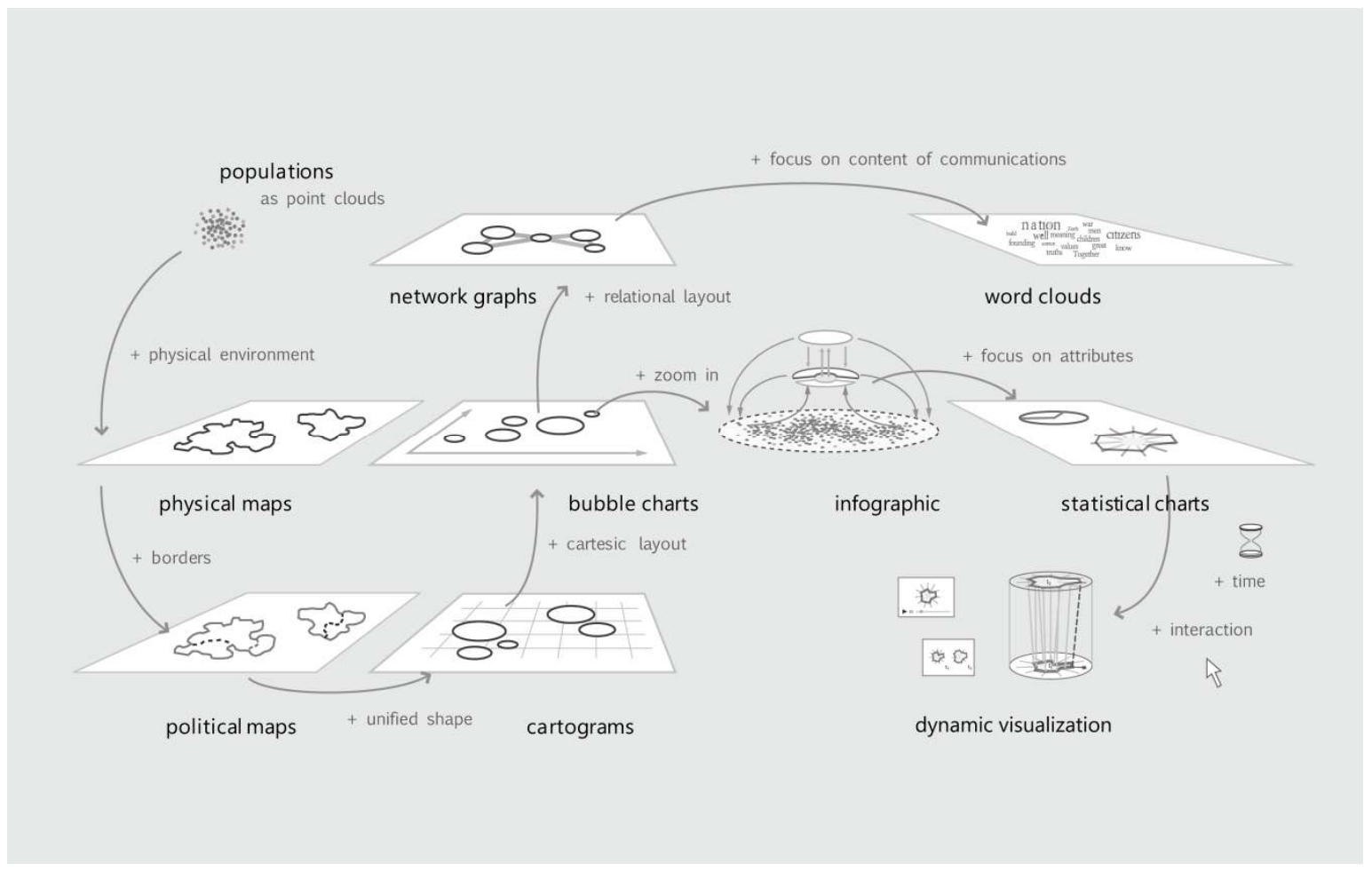

Figure 8: A synoptic perspective on the presented visualization methods, using arrows to show some of the possible conceptual connections and visual-analytical transitions between them.

Figure 8 shows a synoptic arrangement of all the methods introduced so far and presents a selection of possible transitions between them. The annotated arrows signify potential translations from one spatial layout to another and thus deliver a blueprint on how screen-based, interactive multi-method representations could operate in a more synergetic fashion. ${ }^{4}$ In this context, we can see, for example, that the infographic could arise directly out of the circles used by cartograms, bubble charts, or network graphs. These circles, in turn, could be reconstructed as abstractions of the real physical shapes of political territories (left). On the other side, statistical charts (e.g., a piechart showing the parliamentary distribution of votes) can be drawn directly from the infographic. ${ }^{5}$

So beyond simply using visualization methods - and making their operating principles transparent to users - we consider their orchestrated, syntactic use to be a major challenge for our field to offer coherent and connectable insights to the people. However, the challenges lie not only in the development of inter-operable methods; they will also have to be tackled in particular towards the right hand side of the topic-receiver axis (cf. Figure 1), where casual recipients watch their mediaspheres with vastly different backgrounds, preferences and levels of visual literacy.

\section{Dealing with Visual Communication Barriers}

Empirical research into human-computer interaction and the use of visual analytical tools (e.g., BELIV, 2012) emphasizes the fact that, in any visual communication setup, it is ultimately the

\footnotetext{
${ }^{4}$ For an elementary implementation, see the rGapminder interface, where switching between a political map and a scatter plot view on nations allows to follow the layouts' re-arrangement by the means of seamless transitions.

${ }^{5}$ Basically, inter-operable connections allow the building of personalized and scalable visualization systems, where a "you-are-here" (on a map) translates coherently into a "and-that's-happening-over-there" shown, for example, by a line chart. In other words, it takes us from the familiar to the unknown and distant (also in a non-geographic sense). We contend that such a mental-map-guiding visualization environment would allow a new form of (political) data journalism - one for which even the best of the current (single-method-based) state-of-the-art can only provide a sparse impression.
} 
receiver or user (B) who decides whether there will be insights or not. This is determined not only by their level of method literacy, but also by their specific motivations, intentions, and skills, including their ability to effectively operate InfoVis interfaces and tools. If these issues are ignored, users will ironically become the barriers which prevent an intended information transfer. Given the basic tenet of usability design methods, which can be rephrased as: "Know your audience.", this seems to be a challenge which merits systematic investigation, particularly with regard to a broader public. Yet in the field of political InfoVis, barriers to understanding - and corresponding counterstrategies - seem to be rather understudied, leaving information designers without empirical results to work with. In a first step, we would therefore like to assemble suitable findings from the broader research field to extrapolate a picture of the expected users. Among these findings, knowledge about the differences between expert and non-expert users, together with their consequences for the design of InfoVis interfaces for "casual use" (Pousman \& Stasko, 2007) are clearly of high importance.

As prototypical InfoVis users, experts seek to actively explore data to make sense of it. Since data exploration is (part of) their job, they share an intrinsic motivation to explore data and hunt for new insights. They are proven specialists and usually have the broad domain knowledge required to justify an interpretation of the results. Professional visual data analysts are also highly skilled at reading and interpreting graphs, have knowledge of the data source and data collection, and understand the pitfalls when it comes to correctly interpreting results. Non-experts, on the other hand, often have a contrasting profile, based on their typical goals and motivations for exploration, their prior knowledge relating to the data, and their proficiency in the use of data tools. In the following section, we will discuss these aspects in more detail and derive some possible design strategies to support their success in the world of visual data analysis.

\subsection{The Motivations and Goals of Non-Experts in Casual Contexts}

In their study of InfoVis use in casual contexts (i.e. primarily non-professional use in non-work settings), Sprague and Tory (2012) report on the specific motivations of their non-expert participants. Their findings show that these are mainly driven by personal relevance and that their intrinsic motivating factors are to learn something new or acquire a deeper understanding of the subject matter. Utilitarian motives, i.e. learning something practical, similarly encourage the use of visualizations. But not least, casual users of visualizations also simply want to be entertained either by the content or by the aesthetics of the representation itself. Among the extrinsic factors that influence the use of visualizations, procrastination (i.e. the avoidance of boredom) or social pressures (i.e. the demand to be or get informed) played crucial roles. Nonetheless, even laypeople like their observations to add up and make some sense, behavior which Sprague and Tory (ibid.) refer to as "productive relaxation".

Further differences can also be identified with regard to the types of insights that are sought. While analytical insights are the main goal for experts ("the large or small eureka moments where the body of data comes into focus", Pousman et al., 2007, p. 1150), things look different for casual InfoVis users. Laypeople are interested in gaining insights that provide awareness, since these although lacking a reasoning process with a clear conclusion - can also disclose basic patterns in or give the user a feeling for the data. Another area of preference are insights into social life and social situations, along with the actual experience of social interaction and collaboration with user generated content itself (Viegas, Wattenberg, McKeon, Van Ham, \& Kriss, 2008), which might be a particularly relevant aspect for Open Society and Open Data projects. Users also reported that they were interested in gaining reflective insights, e.g. about themselves, the world, and their place in it.

Experts and laypeople also differ in how they gain insights. While expert users actively hunt for insights and are driven by hypotheses, studies showed that laypeople often gather insights in a more passive manner by collecting salient pieces of information (Smuc, Mayr \& Risku, 2010). 


\subsection{The Role of Entertainment and Aesthetic Design}

Traditional strategies to raise the accessibilty of InfoVis interfaces include designing them in line with design guidelines and learning from best practices. A large number of best practices for data visualizations made for research have been published in recent years (Tufte, 1983; Few, 2004). Many of these are characterized by functional aesthetics and advocate a clear and simplistic design; parsimonious use of ink is one of the guiding principles, whereas decorative illustrations (so-called chart junk) are said to inhibit a clear look at the data. While this might hold true for visualizations within a scientific discourse, the mantra of functional design is still being debated for casual InfoVis. Bateman et al. (2010), for example, show that junk charts can facilitate memorization and that an appropriate balance between utility and aesthetic appeal seems necessary. Danziger (2008) proposes that user entertainment and narration are worth considering to effectively establish the context and, thus, facilitate the use of the interface and the learning of domain knowledge. Affective cues can further help to enhance involvement, which is key to making InfoVis appear useful on a personal level.

\subsection{The Role of Usability Design and Interaction Methods}

As with software development in general, a user-centered design approach (Gould \& Lewis, 1985 ) to overcome barriers on the receiver side can also be beneficial when developing and implementing InfoVis for the public sphere. Knowing your audience is probably the most influential strategy for successful development, so an early and continual focus on users during development is essential, as is keeping in mind their goals, motivations, and tasks. However, when developing for the general public, the heterogeneous structure of the audience could prove to be one of the most challenging issues that needs to be tackled. Weather the results from user studies with experts are generalizable and applicable for casual InfoVis still leave many questions open and therefore serves as a rich field for future research (Grammel, 2010). A permanent feature in the user-centered design process is the empirical measurement of the efficiency of InfoVis tools and interfaces. Modern methods to test and accompany the development of information visualizations often use a mix of multiple methods or try to overcome some of the limitations of traditional laboratory usability tests. For example, ethnographic methods (Shneiderman \& Plaisant, 2006; Sprague et al., 2012) in which users are observed during everyday usage of a tool offer the benefits of real world settings and embeddedness in the context of usage. Another decisive factor in design is iterative, cyclic development. Aside from the procedural view, where working with sketches or mock-ups, early prototyping, and repeated redesign phases have now become quasi industry standard, the development of InfoVis for the general public could also benefit from ongoing user participation. A comment function, for example, is a simple to implement asset, and the resulting social exchange could serve not only to raise visual literacy but also to influence the redesign of the tool.

Alongside a user-centered design process, interaction is a potent strategy for relieving visual representations from an overdose of visual clutter and complexity, yet making details visible on demand. Such interaction components have to be selected with special care for non-expert and novice users. Grammel et al. (2010) recommend a tight integration of interaction methods into the InfoVis creation process, and suggest the provision of further semantics (e.g. through mouseovers) as well as searching and filtering functions as possible options. When it comes to working with interactive and changing layouts, animation is a recommended method for providing seamless transitions and thus supporting the preservation of the user's mental map (Friedrich \& Eades 2002). This strategy also proves beneficial when users have to follow changes between different views on the same set of data (cf. section 2.9) (Friere \& Rodriguez, 2006). Another possible approach makes use of case-based representations to support novices. Here, visualizations are developed on the basis of the user's familiarity with certain data and also on the past experiences of comparable user groups (Freyne \& Smyth, 2010). In a similar way, the provision of visual 
metaphors is a well-known technique for raising the accessibility and comprehensibilty of InfoVis tools and interfaces with specific regard to first time and casual users (Danziger, 2008).

\section{Conclusions and Outlook}

In this article, we have examined the current state and future potential of InfoVis methods in the field of political education and communication. Building on a generic communication model, we studied the principles of and possible synergies between selected visualization methods. We then went on to focus on how to overcome familiar communication barriers when addressing non-expert audiences, who constitute the majority in our given field. We consider the following aspects to be of specific relevance, especially with regard such recipients:

- On the sender (A) and recipient (B) sides, the promotion of visual literacy will be a main objective that has to be tackled on a daily basis by providing explanations and introductions to current InfoVis methods and means (cf. section 2), as well as through long-term efforts to advocate and promote multimodal education policies and provide corresponding recommendations and advice to educational boards.

- To complement the development of method literacy, we consider the development and exploitation of inter-method-synergies (cf. Figure 8) to be a decisive challenge and a key task for the future. Only on the basis of such alignments do we expect visual representations to complement verbal reflections with a sufficiently combinable basic vocabulary to reassemble complex representations in a visual modality.

- User-centered design procedures with their extensive repertoire of usability methods to raise the probabilities of communication and education success rates on the recipient side (section 3 ) will need to be patiently enforced and defended. These range from traditional principles like "know your user" and leverage interaction methods to case-based design, narration, affective cues, maintenance of mental map, gamification, etc., and are of particular relevance for non-expert audiences.

- Contradicting or complementing the tenets of existing InfoVis literature, aesthetics and entertainment aspects will be essential when it comes to designing engaging visualizations for recipients in a casual context. We expect traditional design recommendations aimed at the parsimonious use of ink within sober frameworks of functional design to be extended and rewritten for these constellations. While these strategies will remain intact for experts and workoriented settings, we anticipate the emergence of a more persuasive and playful generation of InfoVis interfaces and tools for the casual audience.

We consider these reflections to be of practical relevance in an education context (interactive interfaces, textbook design, didactics), for journalism (print and data), as well as for direct communication efforts by governmental organizations, NGOs, political parties, and public initiatives. As far as the related risks of political spin are concerned, we would like to link up here with the "Critical InfoVis" initiative launched by Dörk et al (2013), who sketch ways of effectively minimizing the hazards of propaganda production within the visual communication realm. From an academic reflection perspective, we hope that this article will contribute to an emerging conceptual discussion and strengthen further developments in and connections between political InfoVis endeavors. Taken as a whole, these developments give adequate reason to expect that hybrid (i.e. hyper-visual-textual) communication methods will offer new ways of representing complex matters of concern (cf. $>$ AIME) and will serve to support multimodal cognition and communication within contemporary news and information spaces. 


\section{References}

Bach, B., Dragicevic, P., Archambault, D., Hurter, C., \& Carpendale, S. (2014). A Review of Temporal Data Visualizations Based on Space-Time Cube Operations. In EuroVis-State of the Art Reports (pp. 23-41).

Bagrow, L. \& Skelton, R. A. (2009). History of Cartography. 2nd Edition. New Brunswick, NJ: Transaction Publishers.

Bateman, S., Mandryk, R. L., Gutwin, C., Genest, A., McDine, D., \& Brooks, C. (2010). Useful junk?: The effects of visual embellishment on comprehension and memorability of charts. In Proceedings of the SIGCHI Conference on Human Factors in Computing Systems (pp. 2573-2582).

BELIV '12 (2012). Proceedings of the 2012 BELIV Workshop: Beyond Time and Errors - Novel Evaluation Methods for Visualization. New York, NY: ACM.

Card, S., Mackinlay, J., \& Shneiderman, B. (1999). Readings in Information Visualization: Using Vision to Think. San Francisco, CA: Morgan Kaufmann Publishers.

Card, S. (2008) Information visualization. In A. Sears and J.A. Jacko (eds.), The Human-Computer Interaction Handbook: Fundamentals, Evolving Technologies, and Emerging Applications , (pp. 509-543). Hillsdale, NJ: Lawrence Erlbaum Assoc Inc.

Danziger, M. (2008). Information Visualization for the People. Thesis. Massachusetts Institute of Technology.

Dörk, M., Feng, P., Collins, C., \& Carpendale, S. (2013). Critical InfoVis: exploring the politics of visualization. In Proceedings of CHl'13 Human Factors in Computing Systems (pp. 2189-2198).

Dorling, D. (2011). Area cartograms: their use and creation. In M. Dodge, R. Kitchin, C. Perkins (Eds.) The Map Reader: Theories of Mapping Practice and Cartographic Representation (pp. 252-260). Hoboken, NJ: Wiley-Blackwell.

Engelhardt, Y. (2006). Objects and spaces: The visual language of Graphics. In: D. Barker-Plummer, R. Cox, and N. Swoboda (Eds.) Diagrammatic representation and Inference (pp. 104-108). Berlin: Springer.

Felten, P. (2008). Visual literacy. Change: The magazine of higher learning 40(6), 60-64.

Few, S. (2004). Show me the numbers: Designing Tables and Graphs to Enlighten. Oakland, CA: Analytics Press.

Freyne, J., \& Smyth, B. (2010). Visualization for the Masses: Learning from the Experts. In I. Bichindaritz \& S. Montani (Eds.), Case-Based Reasoning. Research and Development Vol. 6176 (pp. 111-125). Berlin: Springer.

Freire, M., \& Rodríguez, P. (2006). Preserving the mental map in interactive graph interfaces. In Proceedings of the working conference on Advanced visual interfaces (AVI) (pp. 270-273). New York, NY: ACM.

Friedrich, C., \& Eades, P. (2002). Graph drawing in motion. Journal of Graph Algorithms and Applications, 6(3), 353--370.

Grammel, L., Tory, M., \& Storey, M. (2010). How information visualization novices construct visualizations. Visualization and Computer Graphics, IEEE Transactions on, 16(6), 943-952.

Gould, J. D., \& Lewis, C. (1985). Designing for usability: key principles and what designers think. Communications of the ACM 28(3), 300-311.

Le Monde diplomatique (Ed.). (2012). Atlas der Globalisierung: die Welt von morgen. Berlin: TAZ.

OECD. (2013). How's Life? Measuring Well-being. Paris: Organisation for Economic Co-operation and Development.

Pousman, Z., Stasko, J. T., \& Mateas, M. (2007). Casual information visualization: Depictions of data in everyday life. Visualization and Computer Graphics, IEEE Transactions on, 13(6), 1145-1152.

Risku, H., Mayr, E., Windhager, F., \& Smuc, M. (2011) An Extended Model of Knowledge Communication: The Situational View of Dealing with Asymmetries. Fachsprache XXXIV 3(4), 168-186.

Shneiderman, B., \& Plaisant, C. (2006). Strategies for evaluating information visualization tools: multi-dimensional in-depth long-term case studies. In Proceedings of the 2006 AVI workshop on BEyond time and errors: novel evaluation methods for information visualization (p. 1-7). Venice, Italy: ACM.

Smuc, M., Mayr, E., \& Risku, H. (2010). Is your user hunting or gathering insights?: Identifying insight drivers across domains. In Proceedings of the 3rd BELIV'10 Workshop: BEyond time and errors: novel evaLuation methods for Information Visualization (pp. 49-54).

Sprague, D., \& Tory, M. (2012). Exploring how and why people use visualizations in casual contexts: Modeling user goals and regulated motivations. Information Visualization, 11(2), 106-123.

Tufte, E. R., \& Graves-Morris, P. R. (1983). The visual display of quantitative information. Cheshire, CT: Graphics press.

van Wijk, J. J. (2005). The Value of Visualization, In Visualization'05, Proceedings of the IEEE Conference on (pp. 567574).

Viegas, F. B., Wattenberg, M., McKeon, M., Van Ham, F., \& Kriss, J. (2008). Harry potter and the meat-filled freezer: A case study of spontaneous usage of visualization tools. In Hawaii International Conference on System Sciences, Proceedings of the 41st Annual (pp. 159-159). 
Viegas, F. B., Wattenberg, M., Van Ham, F., Kriss, J., \& McKeon, M. (2007). Manyeyes: a site for visualization at internet scale. Visualization and Computer Graphics, IEEE Transactions on, 13(6), 1121-1128.

Windhager, F. (2013). On Polycubism. Outlining a dynamic information visualization framework for the humanities and social sciences. In M. Füllsack (Ed.). Networking Networks. Origins, Applications, Experiments (pp. 28-63). Vienna, Austria: Turia + Kant.

\section{$\nearrow$ Web Sources and Interactive Visualizations:}

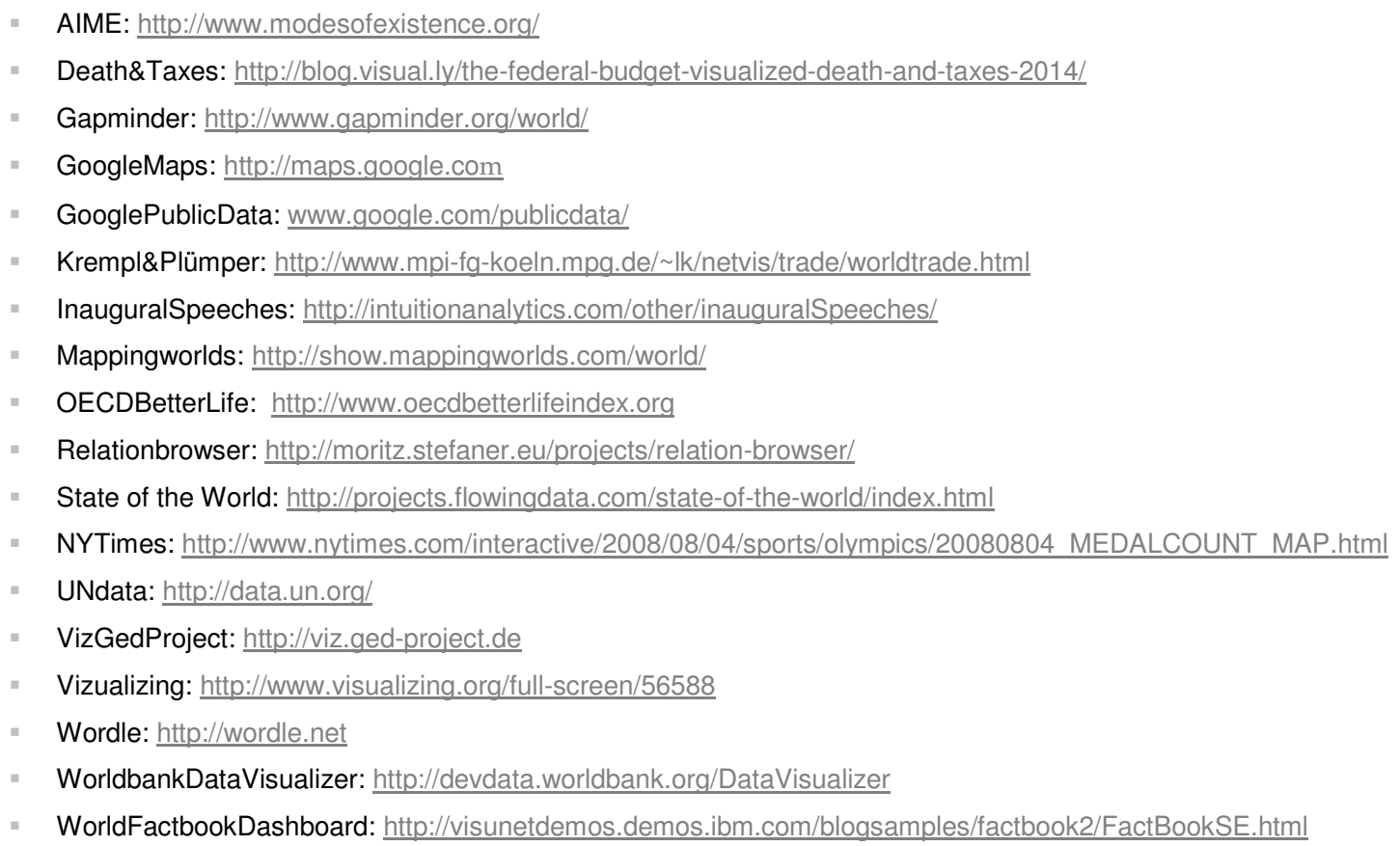

\section{About the Authors}

\section{Florian Windhager}

Florian Windhager graduated in Philosophy from the University of Vienna, Austria. Extracurricular studies on the public communication of science and on cultural and media philosophy at the Academy of Fine Arts and the Academy of Applied Arts in Vienna. Member of the academic staff at the Center for Cognition, Information and Management at the Danube University Krems, Austria since 2007. Research focus: information visualization, social network research, and concept development for visual analytics interfaces. Further information and selected publications: http://www.donauuni.ac.at/florian.windhager.

\section{Michael Smuc}

Michael Smuc graduated in Psychology from the University of Vienna, Austria. He joined the Danube University Krems, Austria in 2007, where he is currently Head of Research at the Center for Cognition, Information and Management. His research interests focus on usability methods, the evaluation of Infoviz-tools, graph comprehension, and dynamic network analysis. Further information and selected publications: http://www.donau-uni.ac.at/michaels.smuc 